\title{
Subject Index Vol. 65, 1997
}

\section{Rathobiol $\varnothing>$ oy}

Actin 113

Acute myeloid leukemia 223

Aging 155

AIDS 210

Alcohol 39

Alkyltransferase 253

Aneurysms 1

Angiogenesis 287

Animal model 271

Anti-anti-idiotype antibody 229

Anti-idiotype antibody 229

AP-1 241

Apoptosis 61, 140,264

Atherosclerosis 1

Autoantibodies 204

Autoimmunity 190,204

Bcl-2 108, 140 Bioengineering fatigue 1 Bladder cancer 129 Breast cancer 108,129,184 c-erbB-2 163

Cancer susceptibility 293

Carcinogenesis 14, 140

Cataract 146

CD3 216

CD29 91

CD44hiCD45RB10 T cell 155

CD4410CD45RBhi T cell 155

CD45RA 91

CD69 216

Cell adhesion kinase 195

- molecules 241

proliferation 39 Cervical cancer 163 Chemical toxicity 14 Chemotaxis 287 Chlamydia trachomatís 229 Choline 264

Chromosome 8 copy number 184 Chromosomes 1, 6, 7, 9-11, 311 Chronic fatigue 51

- syndrome 57 Colorectum 140 Costimulation 69 Cross-linking 301 Cyclophosphamide 26

Cytogenetics 311 Cytokeratin 100 Cytokine 169,287

production 155 Cytoskeleton 69, 177,331 Cytotoxic cells 83

response 331

Deferoxamine 61 Demyelinating disease 190 Development 146

Discoidin domain receptor kinase 
195 Disease progression 169,210 DNA methylation 253

- $\quad$ ploidy 129

Ectasia 1 EGF-R 163 Embryology 146 Endometrium 177 Endophytic/exophytic growth 253

Endothelial cells 241,287 Envelope 123 Epidemic 51 Epitopes 204 Esophageal cancer 39,253

- carcinoma 195

Exoglycolipid 229

Experimental autoimmune encepha-

lomyelitis 190

F-actin 177

Flaky skin mouse mutation 271

Flow cytometry 26

Fluorescence 69

Fluorescent in situ hybridization

184 Forestomach papillomatosís 271 Fusel oil, methylbutanol 39

Gastric dysplasia 271 Gene expression 293 Genetics 311 Glioblastoma 51 Glomerulonephritis

271 Granulocytes 26 GranzymeB 83 Growth factor receptors 75

Heavy metals 241 Hemodynamics 1 Histology 163

- $\quad$ infection 91

HLA-DQB1 210

HLA-DR 91

Hodgkin's disease 129

Human immunodeficient virus 169

- - infection 91

- - type I 210

- $\quad$ mammary epithelial cancer cells

75

Idiotype 229 Image cytometry 129 Immunodeficiency 46 Immunology 169 Immunoreactivity

100 In situ hybridization 83

- $\quad$ vitro kinase assay 301

Infiltrating ductal carcinoma, breast

184 Influenza 69,331 Integrin receptors 177 Interferon- $\gamma 26$ Interleukin 287 Interleukin-1 ß 26 Interleukin-3 26 Interleukin-6 26 Intimal tears 1 Iron 61

Leiomyomas 113 Leiomyosarcomas 113 Lipid peroxidation 14 Lipooligosaccharide 26 Liver 264 - cancer 129 Lung cancer 129 Luzindole 190 Lymph nodes 129 Lymphocyte subsets 91

Lymphoma 61, 83

Mast cells 271 Melanoma 311 Melatonin 190 Memory T cell 155 Meningococcus 26 Metastasis

311 Mismatch DNA repair 293 Mohave 51 Mouse 146, 190 Multivariate analysis 129 Mural

fragility 1 Murine helper T cell 155 Myelocathexis 46 Myelosuppression 26

Naive T cell 155 Natural killer 331 Neural networks 129 Neutralization 229 NF-кB/IкB 241

Nitrosamines 39

OKT3 69

p15E 123p53 108, 140 p56lck 301

Parakeratosis 39, 253 PCNA 108 Perforin 83 Phosphorylation 46 Phytohaemagglutinin 69

Polymerase chain reaction 293 Positive selection 216 Prognosis 129 Proinflammatory mediators 241

Proliferative skin disease 271 Prostate cancer 129 Proto-oncogenes 75 Psoriasis 271 
Receptor antagonist 190 Retrovims, human endogenous 123 Reverse transcription 293 RNA 57 RT-PCR 57

S-phase fraction 129 Septic shock 26 Signal transduction 241,301 Smooth muscle 113 Sodiumproton antiport 46 Squamous cell carcinoma 100

intraepithelial lesion 100 Stage I breast cancer 184

II breast cancer 184 Stealth virus 51, 57 Stenoses 1

Steroid hormone receptor 75

Tcell 190

T-cell-restricted intracellular

antigen-1 83 Tamoxifen 177 Testicular cancer 129 Tetrasomy8 184 Thymoma, human T cell 216

Tortuosity 1 Trachoma 229 Transferrin receptor 61 Transforming growth factor- $\beta 264$

Translocation 223 Trisomy8 184 trkE $195 \operatorname{TrR} \alpha \beta 216$

Tumor progression 311 Tumorigenesis 113 Tyrosine kinase 195

Uterine cervix 100

Vaccine 229 Virology 169 Vitamin E 14

White blood cell lysis 26

Zinc 39

- $\quad$ deficiency 253

\section{KAHGEÍl}

Fax + 41613061234 E-Mail karger@karger.ch www. karger. com

(C) 1998 S.KargerAG, Basel 